\title{
QUELS SONT LES OBJECTIFS NON MEDICAUX INDIVIDUELS ET COLLECTIFS ?
}

\section{WHAT ARE THE NON-MEDICAL INDIVIDUAL AND COLLECTIVE OBJECTIVES?}

\section{Marcel Goldberg}

Professeur de santé publique, Inserm Unité 1018 - Université Versailles Saint Quentin

\section{CONTEXTE : L'ABSENCE D'OBJECTIFS EXPLICITES DU SUIVI POST-PROFESSIONNEL}

Le suivi post-professionnel (SPP) des personnes ayant été exposées à l'amiante durant leur vie professionnelle est un dispositif qui existe depuis 1995, mais dont les objectifs n'ont jamais été clairement énoncés. On peut faire l'hypothèse, à la lecture des textes définissant le SPP [1], qu'il s'agit en premier lieu d'un dispositif qui vise à diagnostiquer précocement les pathologies liées à l'amiante; l'objectif serait donc d'assurer une prévention secondaire (l'exposition ayant déjà eu lieu, les pathologies à venir sont inaccessibles à une prévention primaire). Cet objectif est donc uniquement médical et les objectifs non médicaux individuels et collectifs n'ont pas été explicités.

Par ailleurs, le dispositif tel qu'il est décrit dans les textes n'est pas véritablement opérationnel puisqu'il n'est pas accompagné des procédures permettant de connaître ses bénéficiaires potentiels de façon suffisamment exhaustive : le nombre de SPP « amiante » pris en charge chaque année comparativement aux estimations publiées montre que seule une très faible proportion des personnes potentiellement concernées bénéficient de ce dispositif ${ }^{1}$; ceci est du essentiellement à la difficulté d'obtention des attestations d'exposition qui constituent le mode d'entrée principal dans le SPP, largement expliquée par l'ignorance de l'exposition par les sujets, leur employeur et leur médecin du travail au moment du départ en inactivité. Dans ces conditions, il est donc difficile de rattacher au SPP des objectifs non médicaux, qu'ils soient individuels ou collectifs, le dispositif en lui-même n'étant pas à même de les remplir puisqu'il ne touche qu'une faible minorité de bénéficiaires, et qu'il n'a pas défini de tels objectifs ni évidemment prévu leur évaluation. Dans ce qui suit, ce sont donc les objectifs que devraient poursuivre le SPP qui sont envisagés.

La catastrophe sanitaire liée à l'amiante est connue et bien décrite depuis plusieurs décennies. De nombreux rapports scientifiques [2,3] et parlementaires [4-6] ont fait un état des lieux très complet de la situation en France. Cette catastrophe se singularise par plusieurs aspects. D'une part, elle est de grande ampleur: près de 100000 décès imputables à l'amiante sont attendus dans les 25 prochaines années. Cela s'explique par la très large utilisation de l'amiante dans de nombreux secteurs d'activité et professions. On estime ainsi que $25 \%$ des hommes salariés aujourd'hui retraités ont été exposés à l'amiante $[7,8]$, ce pourcentage étant deux fois plus élevé parmi les hommes artisans retraités [9]. D'autre part, la gestion du risque lié à l'utilisation de l'amiante, connu et documenté depuis les années 1970, a souffert de nombreux dysfonctionnements conduisant à un important retard dans la mise en œuvre des premières mesures de prévention, accentuant encore l'ampleur de la catastrophe.

La reconnaissance de cette gestion défaillante a incité l'État à mettre en œuvre des procédures spécifiques de réparation du préjudice dans les années 1990 : le suivi postprofessionnel (SPP) en 1995, puis le dispositif de départ en retraite anticipé (FCAATA) et un dispositif d'indemnisation intégrale (Fonds d'indemnisation des victimes de l'amiante : Fiva). Si les deux derniers dispositifs visent exclusivement la réparation du préjudice, le SPP poursuit plutôt un objectif de prévention secondaire. II s'agit en effet d'un dispositif de dépistage ciblé des pathologies en lien avec l'amiante ; les objectifs médicaux apparaissent donc au premier plan.

\footnotetext{
${ }^{1}$ Bien que la CNAMTS ne publie aucun chiffre sur le nombre de SPP pris en charge, ni a fortiori sur le nombre de demandes, il semble qu'environ de 3 à 5000 SPP "Amiante » soient accordés chaque année.
} 
Néanmoins, il est clair le SPP est susceptible d'apporter aussi des bénéfices individuels et collectifs non médicaux qui, dans le contexte particulier de l'amiante, présentent une importance particulière du fait que l'exposition à un cancérogène avéré a été de facto imposé par un tiers aux personnes concernées.

On abordera aussi dans ce texte le point de l'impact psychologique potentiel du SPP, qui a parfois été évoqué.

\section{OBJECTIFS NON MEDICAUX INDIVIDUELS}

Information. Le premier bénéfice non médical implicitement visé par le SPP est d'apporter une double information des personnes: sur leur exposition professionnelle passée à l'amiante et sur leurs droits à bénéficier d'un SPP. Le devoir d'équité impose que cette information soit proposée à toute personne susceptible d'avoir été exposée à l'amiante, comme cela est précisé dans la loi $n^{\circ}$ 2005-1579 de financement de la sécurité sociale pour 2006 du 19 décembre 2005, qui prévoit dans son article 81 que «toute personne qui, du fait de son activité professionnelle, est susceptible d'avoir été exposée à l'inhalation de poussière d'amiante est informée par sa Caisse primaire d'assurance maladie dans des conditions précisées par décret de son droit de bénéficier gratuitement de la surveillance médicale post-professionnelle, visée à l'article D. 461-25 du code de la sécurité sociale, incluant le cas échéant, les examens médicaux complémentaires appropriés. " [10]. Le Comité consultatif national d'éthique, consulté lors de la mise en place de la phase pilote du programme Spirale, a également confirmé le devoir d'information des personnes exposées à l'amiante ${ }^{2}$.

Cette information doit être pertinente au niveau individuel, c'est-à-dire accompagnée d'éléments sur le niveau et la durée d'exposition, sur les risques pour la santé, en particulier en cas d'exposition conjointe au tabac, et sur les dispositifs de prise en charge. Comme l'exposition à l'amiante est souvent méconnue des personnes concernées, cette information doit être proposée à l'ensemble des personnes susceptibles d'avoir été exposées.

Actuellement, le SPP rempli très mal cet objectif, puisque l'accès au SPP nécessite en premier lieu une démarche active de la personne, ce qui implique de facto la connaissance de son exposition, ce qui n'est souvent pas le cas : ainsi, les résultats de la phase pilote du programme Spirale montrent qu'environ la moitié des retraités ayant été exposés à l'amiante l'ignorent [11]. De plus, l'accès est largement conditionné par le fait de posséder l'attestation d'exposition signée de l'employeur et du médecin du travail. Cette attestation ne peut être délivrée que par le dernier employeur, souvent différent de l'employeur lors de l'exposition du salarié. En pratique, seuls les salariés de certaines grandes entreprises, y ayant fait l'ensemble de leur carrière, bénéficient de cette attestation, ce qui ne représente qu'une faible part des exposés, la grande majorité des expositions ayant eu lieu dans les PME-TPE $[8,11]$. Cet état de fait contribue à renforcer l'inéquité : en effet, d'une part on sait que les mesure de protection contre les nuisances professionnelles sont dans l'ensemble moins bien appliquées dans les PME-TPE, et d'autre part les personnes y ayant travaillé ont moins de chances de se voir remettre une attestation d'exposition leur ouvrant la possibilité d'un SPP.

On peut donc considérer qu'un premier objectif non médical à visée individuelle est d'apporter aux personnes concernées une information sur leur exposition professionnelle passée à l'amiante et sur leurs droits à bénéficier d'un SPP.

Reconnaissance sociale. Le second bénéfice individuel non médical visé par le SPP est d'améliorer la reconnaissance sociale du risque. Les personnes exposées à l'amiante l'ont été le plus souvent sans en avoir connaissance, sans être informées des risques sanitaires pourtant largement connus et parfois sans mise en œuvre de moyens de protection. Face au retard pris en matière de prévention de ce risque de la part de l'État et des employeurs, et à

\footnotetext{
${ }^{2}$ Courrier adressé en janvier 2005 par le Comité consultatif national d'éthique aux responsables du programme Spirale, reproduit en annexe.
} 
l'importance de la morbidité et mortalité induite par l'amiante, il existe une exigence morale de reconnaissance du préjudice subi par les personnes exposées à l'amiante, comme l'a souligné le Comité consultatif national d'éthique dans le courrier déjà cité. L'enquête de " satisfaction » menée auprès des personnes ayant été incluses dans le programme Spirale montre que celles-ci sont très sensibles à cet aspect [11]. Ceci passe en premier lieu par la reconnaissance "officielle» de la réalité de l'exposition; l'accès au SPP offre cette reconnaissance.

Au total, alors que le principe d'équité voudrait que toute personne ayant été exposée se voit proposer la possibilité d'un SPP lors de la prise de retraite, seule une minorité des retraités ayant été exposés est en position d'en bénéficier, alors que les exclus du dispositif se comptent essentiellement parmi ceux qui ont été le moins protégés pendant leur vie active et qui disposent d'une moins bonne information.

Dans ce domaine, il faut souligner un autre facteur contribuant à l'inéquité vis-à-vis d'un bénéficie potentiel : certaines CPAM exigent l'attestation d'exposition pour délivrer le SPP, tandis que d'autres acceptent des éléments indirects comme des certificats de travail ou l'expertise fournie dans le cadre de Spirale [11]. Ainsi, selon l'attitude de leur CPAM et sans autre justification, certaines personnes se voient, à exposition comparable, accorder ou refuser le SPP ; dans ce cas, qui revient à contester une exposition même réelle, on est face à un déni de reconnaissance sociale.

\section{Un second objectif non médical à visée individuelle du SPP est donc d'assurer une reconnaissance sociale aux personnes ayant été exposées à l'amiante durant leur carrière.}

Accès aux dispositifs de réparation. Enfin, le SPP a aussi pour bénéfice non médical individuel indirect de faciliter l'accès aux dispositifs de réparation financière en cas de survenue ultérieure d'un problème de santé lié à l'exposition à l'amiante. Comme pour le SPP, l'accès aux dispositifs de réparation nécessite une démarche volontaire de la part de la personne ou de ses ayants-droits, ce qui implique de connaître d'une part son exposition et son rôle possible dans la survenue d'une pathologie, et d'autre part l'existence des dispositifs. Le fait de bénéficier du SPP facilite donc l'accès à la réparation puisqu'à la fois la personne et son médecin seront informés de l'exposition, de ses risques et des dispositifs de réparation. Ceci est confirmé par l'étude expérimentale SPP-A menée de 2002 à 2005 à la demande de la CNAMTS et de la DGT dans quatre régions : une déclaration de maladie professionnelle a été faite par $8,7 \%$ des sujets inclus dans l'expérimentation, ce pourcentage se montant à $15,4 \%$ parmi ceux qui ont bénéficié d'un examen tomodensitométrique; la très grande majorité de ces demandes ont fait l'objet d'une acceptation, puisque seulement $6,9 \%$ ont été refusées, la plupart pour non respect des conditions prévues aux tableaux de maladies professionnelles [12]. Le Fiva, de son côté, indique dans son huitième rapport d'activité que 6563 nouveaux dossiers de victimes ont été répertoriés en 2008 [13].

Concernant l'accès aux dispositifs de réparation financière, il faut souligner que la plupart des médecins traitants, en première ligne de la prise en charge des patients, ignorent la possibilité d'une étiologie potentielle des cancers du poumon liée à l'amiante chez leurs patients, ainsi que les formalités à accomplir pour une demande de maladie professionnelle, comme l'a montré une récente étude dans la région Provence-Alpes-Côte-d'Azur [14]. Ceci obère les chances d'une indemnisation, puisque le médecin est habituellement l'informateur privilégié de son patient, et que le certificat médical indispensable pour une demande maladie professionnelle ou d'indemnisation par le Fiva doit être établi par le médecin. Par ailleurs, lorsque le patient fait la démarche, l'instruction du dossier de maladie professionnelle ou d'indemnisation par le Fiva est facilitée puisque la réalité de l'exposition a déjà été établie.

Au total, le fait de bénéficier d'un SPP augmente donc beaucoup les chances d'indemnisation pour une personne ayant été exposée et présentant un problème de santé 
lié à l'amiante. Bien qu'aucune étude n'ait été réalisée pour analyser ce point, on peut faire l'hypothèse que l'existence du SPP "Amiante » a certainement contribué à la forte augmentation du nombre de pathologies dues à l'amiante indemnisées observée depuis une décennie (cf. plus loin).

Un autre bénéfice potentiel concerne la cessation anticipée d'activité, ouverte dès l'âge de 50 ans aux personnes atteintes de maladies professionnelles liées à l'amiante. Le bien-fondé de ce dispositif n'a fait, à ma connaissance, l'objet d'aucune étude, bien qu'il soit vraisemblable que pour les pathologies invalidantes, accompagnées d'un pronostic péjoratif, il soit bénéfique en termes de santé et sans doute en termes psychologiques. II serait nécessaire de mettre en place des études spécifiques pour analyser cette question, en soulignant que de telles études seraient méthodologiquement complexes si on veut mettre en évidence un bénéfice spécifique de cette mesure chez des sujets atteints d'une pathologie en raison notamment de nombreux facteurs de confusion potentiels.

\section{Un autre bénéfice non médical à visée individuelle du SPP est donc de faciliter l'accès aux dispositifs de réparation.}

\section{OBJECTIFS NON MEDICAUX COLLECTIFS}

Les bénéfices collectifs non médicaux découlent largement des bénéfices individuels.

Visibilité sociale. Le premier bénéfice collectif non médical généré par le SPP est la visibilité sociale du problème de l'amiante. II existe en effet un hiatus très important entre les données issues de la littérature scientifique qui recueillent un large consensus, et celles des organismes chargés officiellement des problèmes de la santé au travail et de leur prise en charge. Les données issues de ces derniers font apparaître une forte sous-estimation du risque amiante.

Ainsi, en 2003, moins de 3000 personnes bénéficiaient du SPP pour l'amiante alors que près d'un salarié sur 4 (et un artisan sur 2) a été exposé à l'amiante au cours de sa carrière professionnelle $[8,9,11]$. Comme on l'a indiqué, aucune statistique officielle n'est disponible, mais il semble que ce chiffre ait peu évolué depuis. Ces différences sont dues à la fois aux difficultés à faire reconnaître une exposition ou une pathologie professionnelle liées à des démarches difficiles et au manque d'informations sur ces dispositifs, malgré l'obligation légale faite aux CPAM d'informer les personnes potentiellement exposées rappelée plus haut. L'information individuelle reçue lors de l'accès au SPP devrait donc contribuer à l'utilisation des dispositifs de réparation, et ainsi à la visibilité du problème de l'amiante. Rappelons que dans le système de gestion des risques professionnels, la prévention repose largement, à travers les cotisations, sur la morbidité reconnue. Accroître la visibilité des expositions professionnelles reconnues à des cancérogènes et des pathologies professionnelles contribue donc indirectement à l'amélioration de la prévention.

Dans cette optique, il est intéressant de souligner que le nombre de cas de cancers du poumon indemnisés, qui était très faible voici environ une décennie [15], augmente régulièrement depuis, puisque de 458 en 1999, il était de 808 en 2004 et de 1161 en $2006^{3}$ portant ainsi de $27 \%$ à $66 \%$ la proportion du nombre estimé de cas de cancer du poumon indemnisés. De même, le Fiva enregistre environ 8 à 10000 nouvelles demandes chaque année. II est clair que cette tendance à une meilleure indemnisation des victimes de l'amiante est à mettre largement au compte d'une visibilité sociale accrue des risques dus à l'amiante, comme le montre indirectement le fait que pour les cancers d'origine professionnelle induits par d'autres agents cancérogènes, on observe par contraste une quasi stagnation du nombre des indemnisations, alors même que leur sous-indemnisation est très importante. La connaissance des risques liés à l'amiante qui s'est largement répandue auprès du public, du corps médical et des pouvoirs publics en est très certainement la cause, alors que la connaissance d'autres risques reste trop méconnue. Le

\footnotetext{
${ }^{3}$ Source : CNAM-TS DRP, mars 2009.
} 
développement du SPP auprès d'un plus grand nombre de bénéficiaires potentiels est certainement un élément qui contribuerait à augmenter encore la visibilité sociale du problème de l'amiante, et par là même celle plus générale des cancers d'origine professionnelle.

Dans cette optique, il serait certainement important de relayer au mieux et à diverses échelles institutionnelles (pouvoirs publics, corps médical, partenaires sociaux...), et territoriales les données issues du SPP, qui peuvent constituer notamment à l'échelle locale (municipalités départements) et régionale un support mobilisateur intéressant.

\section{Un objectif non médical à visée collective du SPP est donc d'améliorer la visibilité sociale du problème de l'amiante et celle de l'ensemble des cancers d'origine professionnelle.}

Équilibre des comptes de l'Assurance maladie. II faut considérer que la prise en charge financière des soins médicaux pour les pathologies non reconnues comme maladies professionnelles est assurée par la branche maladie de la Sécurité sociale, abondée par les cotisations des salariés et des employeurs, alors que c'est la branche AT-MP, financée uniquement par les employeurs, qui assure la prise en charge des maladies professionnelles. La sous-indemnisation des maladies d'origine professionnelle est donc à l'origine d'une situation qui est non seulement inéquitable, mais qui de plus déséquilibre les comptes de l'Assurance maladie au détriment des salariés. La Commission instituée par l'article L. 176-2 du code de la sécurité sociale, chargée de remettre régulièrement au Parlement et au Gouvernement un rapport évaluant le coût réel pour la branche maladie de la sous-déclaration des accidents du travail et des maladies professionnelles, estime qu'un coût situé dans une fourchette de $222 \mathrm{M} €$ à $563 \mathrm{M} €$ hors dépenses d'indemnités journalières pour l'ensemble des cancers (dont la très grande majorité sont des cancers induits par l'exposition à l'amiante) pris en charge en 2005 par la branche maladie de la Sécurité sociale, "peut être légitimement considéré comme engagé au titre des risques professionnels » [16]. Ainsi, le SPP, qui on l'a vu, concourt à améliorer l'accès aux dispositifs de réparation pour les personnes atteintes d'une pathologie liée à l'amiante, est un des éléments qui permettrait un meilleur équilibre des comptes de l'Assurance maladie.

\section{Un objectif non médical à visée collective du SPP est donc de contribuer à un meilleur équilibre des comptes de l'Assurance maladie.}

Un aspect différent de l'impact financier du SPP concerne les modalités de la surveillance médicale au cours du suivi, qui pourraient induire une surconsommation d'examens. On dispose de très peu de données permettant de vérifier si un tel effet existe effectivement. À ma connaissance, la CNAMTS qui prend en charge ces examens pour les sujets relevant du Régime général, n'a jamais réalisé d'étude à ce sujet, bien qu'elle dispose maintenant d'un important recul pour de nombreux bénéficiaires du dispositif. Les données recueillies lors de l'inclusion dans les programmes SPP-A et Espri ne permettent pas d'en juger, car les examens réalisés à cette occasion ont été prescrits dans le cadre du SPP, et seul un suivi à long terme des sujets inclus dans un SPP pourrait apporter des informations utiles dans ce sens. II faut souligner les difficultés méthodologiques de telles études, notamment car les sujets concernés et leur médecin sont normalement tenus de respecter les modalités de suivi médical s'ils veulent bénéficier de la prise en charge financière des examens. Je signale que les programmes expérimentaux Spirale et Espri ont mis en place un dispositif d'évaluation du suivi qui comprend notamment l'analyse des examens réalisés identifiés dans les bases de données de l'Assurance maladie.

Amélioration des connaissances épidémiologiques. Le SPP doit aussi pouvoir contribuer aux activités de surveillance des risques professionnels, en améliorant les connaissances sur les circonstances des expositions, sur les métiers et les secteurs particulièrement touchés, grâce au recueil des historiques professionnel et d'exposition qu'il permet. II est en effet indispensable de disposer, couplé au SPP, d'un recueil de données descriptives fines sur l'exposition vie entière (période, durée, tâches spécifiques, métiers et secteurs d'activité...). 
De telles données permettent de documenter, à l'échelle de la population, les circonstances d'exposition et ainsi de guider en amont la prévention. Les données descriptives provenant de l'étude Espaces, première expérimentation de SPP à l'échelle populationnelle ont permis à l'époque de donner pour la première fois une image précise concernant les métiers et les secteurs exposés à l'amiante parmi les salariés retraités en France [8] ; ces données ont été actualisées par le programme Spirale [11], et étendues aux artisans retraités grâce au programme Espri [9].

Les cohortes longitudinales constituées à partir des personnes ayant bénéficié d'un SPP contribueront quant à elles à apporter des réponses à diverses questions scientifiques qui restent ouvertes, telles que les effets à long terme de l'amiante sur les cancers du larynx et du colon, le devenir des plaques pleurales et des nodules pulmonaires isolés, ou les relations entre asbestose et risque de cancer. Actuellement, les cohortes ARDCO et SPPAmi2, qui sont issues de l'expérimentation menée dans quatre régions, sont en cours de suivi et ont déjà donné des premiers résultats, et les cohortes Spirale et Espri ont été constituées.

Enfin, en termes d'évaluation, un suivi des bénéficiaires du SPP des cohortes Spirale et Espri a déjà été mis en place dans les bases de données de l'Assurance maladie afin de mieux éclairer les enjeux de ce dispositif en termes de bénéfices médicaux et sociaux.

Pour conclure cette partie concernant les connaissances épidémiologiques, il faut rappeler qu'actuellement, il n'existe aucune statistique systématique sur les SPP demandés et acceptés, alors même que ce dispositif fait l'objet d'une prise en charge médicoadministrative ; un recueil et une analyse régulière de données sur le SPP à différents échelons territoriaux (départemental, régional et national) est indispensable pour évaluer régulièrement ce dispositif.

\section{Un objectif non médical à visée collective du SPP est de contribuer à l'amélioration des connaissances épidémiologiques.}

\section{IMPACT PSYCHOLOGIQUE}

L'impact psychologique défavorable de l'annonce d'une exposition passée à l'amiante a parfois été mis en avant pour mettre en cause le principe même du SPP. II s'agit en effet de découvrir une exposition à l'amiante pour des sujets dont la plupart sont a priori non demandeurs d'informations concernant les expositions potentiellement dangereuses qu'ils auraient pu subir. L'annonce d'une exposition passée à un cancérogène comme l'amiante peut avoir des conséquences psychologiques défavorables en termes d'anxiété, alors même que la grande majorité des personnes exposées l'a été à des niveaux faibles et ne développera jamais de pathologie liée à l'amiante, et que le bénéfice en terme de réduction de morbidité ou mortalité d'une prise en charge médicale des personnes ayant été exposées à l'amiante n'est pas établi.

Cependant, les rares travaux empiriques qui ont étudié ce problème ne confirment pas son importance. Une récente étude finlandaise associée à un dépistage par tomodensitométrie de pathologies respiratoires chez des sujets exposés à l'amiante montre au contraire une réduction significative de l'anxiété après l'examen, et qu'un an après celui-ci on n'observe pas de différence pour les variables psychologiques entre ceux pour lesquels les résultats ont été donnés immédiatement et ceux pour lesquels des examens complémentaires ont été nécessaires en raison de résultats positifs ; les auteurs concluent que la procédure de suivi a été bien acceptée et n'a pas généré à long terme d'anxiété excessive ni d'autres effets psychologiques négatifs [17].Une revue de littérature montre que quelques cas d'état de stress post-traumatique ont été rapportés, mais ils ont été observés principalement chez des collègues de patients décédés de mésothéliome. La majorité des travaux publiés mettent en évidence des effets psychologiques modérés chez les sujets informés, qui se manifestent essentiellement sous la forme de réactions anxieuses. II apparaît que les réactions psychologiques et la perception du risque dépendent plus du fait d'avoir été exposé à 
l'amiante que du fait d'être malade, et que les sujets exposés ont des manifestations plus importantes devant une perturbation de leur état de santé, même sans lien avec l'amiante [18].

Deux programmes français de SPP ont tenté d'évaluer l'impact psychologique de cette procédure.

Le programme expérimental SPP-A incluait en Normandie un volet sur ce thème, basé sur l'échelle "Psychological Consequences Questionnaire » (PCQ). Seuls les résultats de la passation de ce questionnaire au moment de l'inclusion ont été rapportés. Ils montrent que le score est plus fréquemment défavorable chez les sujets qui ont été exposés à l'amiante (qu'il s'agisse d'une auto évaluation ou du résultat de l'expertise des expositions) que chez les non exposés, ainsi que chez les sujets qui pensent que l'amiante entraîne des conséquences graves pour la santé, notamment des cancers [19]. On ne sait pas si les sujets ont été suivis pour l'aspect psychologique au-delà de l'inclusion, comme cela avait été prévu dans le protocole initial, ce qui ne permet pas de juger de la persistance de troubles à long terme chez les sujets ayant un score défavorable. Les résultats rapportés sont cohérents avec la littérature internationale : les personnes ayant été exposées sont plus inquiètes que les autres, ceci d'autant plus qu'ils pensent que l'amiante est dangereux pour la santé.

Une post-enquête auprès des participants de la phase expérimentale du programme Spirale a montré que $92 \%$ se déclarent non surpris par l'annonce de l'exposition, et que seuls $2 \%$ se déclarent très inquiets. De même, aucun signalement de troubles psychologiques n'est revenu des Centres d'examens de santé, qui ont reçu environ 4000 personnes dans le cadre du SPP.

Au total, tous les travaux scientifiques soulignent que les conséquences psychologiques du SPP sont très limitées et vraisemblablement du même ordre d'importance que pour tous les dépistages. II semble que le fait d'être, au moment de la prise de contact avec les sujets, informé qu'on a pu être exposé suscite de l'inquiétude chez un nombre limité de sujets, mais que la prise en charge par un programme de SPP a un effet rassurant, même lorsque des anomalies nécessitant des investigations complémentaires sont détectées, et que l'éventuel effet anxiogène s'estompe rapidement avec le temps. La qualité de l'information initiale donnée aux personnes contactées est certainement un aspect important, qu'il s'agisse de l'information lors du contact initial, et sans doute encore plus lors des étapes suivantes, où le rôle des médecins peut être déterminant.

II faut souligner que les données disponibles sur le sujet de l'impact psychologique sont très parcellaires. On ne dispose notamment, à ma connaissance, d'aucune étude concernant l'impact psychologique potentiel associé aux conséquences «sociales» potentielles (demande de bénéficier d'une indemnisation ou d'autres dispositifs) de la découverte d'une pathologie à l'occasion du SPP, bien qu'on n'ait pas de raison de penser que cet impact pourrait être différent si la découverte d'une pathologie ouvrant droit à un bénéfice a lieu à l'occasion d'un SPP ou dans d'autres circonstances. Finalement, malgré les éléments montrant un effet psychologique très modéré, il pourrait être utile de prévoir dans les programmes de SPP la mise en place d'un soutien spécifique pour les quelques personnes qui présenteraient des difficultés psychologiques, comme le recommandent les auteurs de l'étude SPP-A.

II faut également mettre le problème de l'impact psychologique potentiel du SPP en perspective des pratiques pendant la période d'activité professionnelle. En effet, les salariés exposés à l'amiante sont soumis à la "Surveillance médicale spéciale des salariés exposés à l'amiante » ; il s'agit d'une obligation de l'employeur et du médecin du travail qui concerne le plus souvent, comme dans le cas du SPP, des personnes qui ne présentent pas d'altération de la santé, qui sont donc informées de leur exposition et des risques pour la santé, alors que la plupart ignoraient leur exposition avant que le médecin du travail les en 
informe. On peut faire l'hypothèse que pour une procédure identique de suivi médical, l'impact psychologique induit dans un contexte postprofessionnel (période d'inactivité) sera le même que celui induit lors du contexte professionnel (période d'activité). Ce même parallèle peut être fait pour l'obligation de remise d'une attestation d'exposition par l'employeur au moment du départ à la retraite (bien que cette obligation soit peu suivie dans les faits). On peut donc être surpris que le problème d'un éventuel effet psychologique négatif ne soit évoqué que pour le SPP, alors qu'il ne semble pas être mis en cause dans le cadre de la médecine du travail, qui découle pourtant quant à lui d'une procédure réglementaire obligatoire, alors que le SPP repose sur une démarche volontaire des personnes.

Enfin, il faut rappeler que le Comité Consultatif National d'Éthique pour les Sciences de la Vie et de la Santé, consulté à propos de Spirale sur l'aspect éthique d'une procédure active d'identification de personnes ayant été exposées, souligne dans sa réponse déjà citée plus haut que "le bénéfice en terme de reconnaissance facilitée des maladies professionnelles et d'information des retraités dépasse les aspects négatifs, et que s'il y avait un problème éthique il résiderait dans l'indifférence vis-à-vis des retraités exposés ». 


\section{REFERENCES}

1- Arrêté du 28 février 1995. JORF n69 du 22 mars 1995, page 4474.

2- Effets sur la santé des principaux types d'exposition à l'amiante. Expertise collective Inserm. Éditions Inserm 1997.

3- Surveillance épidémiologique des effets de l'exposition à l'amiante : actualités françaises. BEH Numéro thématique - 41-42; 23 octobre 2007.

4- Amiante : quelle indemnisation pour les victimes ?" Rapport d'information $n^{\circ} 301$ (20042005) de M. Gérard Dériot, fait au nom de la commission des affaires sociales, déposé le 15 avril 2005, Paris, Sénat, 2005

5- Rapport d'information de la commission des affaires sociales sur la prise en charge des victimes de l'amiante. Assemblée nationale, 18 novembre 2009.

6- Rapport de la Mission d'information sur les risques et les conséquences de l'exposition à l'amiante. Assemblée nationale, 22 février 2006.

7- Goldberg M, Banaei A, Goldberg S, Auvert B, Luce D, Guéguen A. Past occupational exposure to asbestos among men in France. Scand J Work Environ Health,2000,26:52-61.

8- Imbernon E, Goldberg M, Spyckerelle $Y$ et al. Enquête pilote ESPACES : identification et suivi médical post-professionnel des salariés retraités ayant été exposés à l'amiante. Place et rôle des Centres d'examen de la santé des CPAM. Avril 2001, Saint Maurice, Institut de Veille Sanitaire.

9- Rolland P, Homere J. Programme de surveillance post-professionnelle des artisans ayant été exposés à l'amiante (Espri). Rapport intermédiaire de la phase pilote. Période septembre 2005 - InVS, Février 2007.

10- Loi de financement de la sécurité sociale 2005-1579 du 19 décembre 2005.

11- Spirale - Surveillance post-professionnelle des travailleurs exposés. Rapport intermédiaire de la phase pilote (2ème vague), Inserm Unité 687, Juin 2009.

12- Suivi post-professionnel Amiante - Étude expérimentale. Rapport final, février 2007.

13- Fonds d'indemnisation des victimes de l'amiante - Fiva. 7ème rapport d'activité au Parlement et au Gouvernement. Année 2007 (http://www.fiva.fr/pdf/rapport-fiva-07.pdf). 8ème rapport d'activité au Parlement et au Gouvernement. Année 2008 (http://www.fiva.fr/pdf/rapport-fiva-2008.pdf)

14- Verger P, Arnaud S, Ferrer S, Iarmarcovai G, Saliba ML, Viau A, Souville M. Inequities in reporting asbestos-related lung cancer: influence of smoking stigma and physician's specialty, workload and role perception. Occup Environ Med. 2008;65:392-7.

15- Imbernon E. Estimation du nombre de certains cancers attribuables à des facteurs professionnels. InVS, 2002.

16- Rapport de la commission instituée par l'article L. 176-2 du code de la sécurité sociale. Juin 2005 (http://www.securite-sociale.fr/communications/rapports/diricq/05 diricq.pdf).

17- Vierikko T, Kivistö S, Järvenpää R, Uitti J, Oksa P, Virtema P, Vehmas T. Psychological impact of computed tomography screening for lung cancer and occupational pulmonary disease among asbestos-exposed workers. European Journal of Cancer Prevention: 2009 ; $18: 203-206$.

18- Maurel M, Berna V, Letourneux M, Paris C. Exposition professionnelle ancienne à l'amiante. Conséquences psychologiques de la surveillance médicale et de l'information sur les risques pour la santé. Arch Mal Prof Env. 2006; 67: 7-13. 
19- Maurel M, Stoufflet A, Thorel L, Berna V, Gislard A, Letourneux M, Pairon JC; National Network of Asbestos Post-Exposure Survey, Paris C. Factors associated with cancer distress in the Asbestos Post-Exposure Survey (APEXS). Am J Ind Med. 2009;52:288-96. 